\title{
Linfonodo axilar como sentinela de neoplasia mamária em cadelas ${ }^{1}$
}

\begin{abstract}
Simone P. Bianchi²*, Cristiano Gomes², Saulo P. Pavarini², Verônica S. Mombach², Fabiane R. Santos ${ }^{2}$, Luciane C. Vieira ${ }^{3}$, Luciana O. Oliveira ${ }^{3}$ e Emerson A. Contesini ${ }^{2}$

ABSTRACT.- Bianchi S.P., Gomes C., Pavarini S.P., Mombach V.S., Santos F.R., Vieira L.C., Oliveira L.O. \& Contesini E.A. 2018. [Axillary lymph node as sentinel for mammary neoplasia in bitches.] Linfonodo axilar como sentinela de neoplasia mamária em cadelas. Pesquisa Veterinária Brasileira 38(4):692-695. Faculdade de Veterinária, Universidade Federal do Rio Grande do Sul, Avenida Bento Gonçalves 9090, Bairro Agronomia, Porto Alegre, RS 90540-000, Brazil. E-mail: simonepbianchi@gmail.com

Mammary tumors research in bitches is important due to their high incidence. The aim of this study was to evaluate the importance of the axillary lymph node as a sentinel lymph node for mammary neoplasms in female dogs. Forty-nine bitches with mammary neoplasia were submitted to total unilateral mastectomy, and the axillary lymph node was identified using the patent blue dye. This lymph node was processed routinely for histopathological analysis and stained with hematoxylin-eosin and by immunohistochemistry (IHC) with cytokeratin antibody (AE1/AE3) to search for metastasis. Eight dogs had axillary lymph node metastases, seven of which were detected by histopathology and by IHC and only one by IHC (micrometastasis). One dog who presented tumor in caudal and inguinal abdominal mammary glands had metastases in the axillary and inguinal lymph nodes. It is concluded that the mammary tumor can cause alteration in lymphatic drainage leading to metastases in lymph nodes which normally do not drain certain glands; so the removal of the axillary lymph node should be included as a routine technique to allow better staging of mammary neoplasms of bitches.
\end{abstract}

INDEX TERMS: Axillary lymph node, mammary, neoplasia, bitches, sentinel lymph node, pathology.

RESUMO.- Linfonodo axilar como sentinela de neoplasia mamária em cadelas. 0 estudo dos tumores de mama em cadelas é de grande importância devido à alta frequência com que surgem na clínica de pequenos animais. 0 presente estudo teve como objetivo avaliar a importância do linfonodo axilar como sentinela em neoplasias mamárias de cadelas. Foram avaliadas 49 fêmeas com neoplasia mamária, submetidas à mastectomia unilateral total, utilizando o corante azul patente para a identificação do linfonodo axilar, o qual foi submetido à análise histopatológica com a coloração de hematoxilina-eosina e imuno-histoquímica (IHQ) com anticorpo citoqueratina (AE1/AE3) para procura de metástase. Oito cadelas apresentaram metástases em linfonodo axilar, sendo sete

\footnotetext{
${ }^{1}$ Recebido em 9 de junho de 2017.

Aceito para publicação em 22 de julho de 2017.

${ }^{2}$ Faculdade de Veterinária, Universidade Federal do Rio Grande do Sul (UFRGS), Avenida Bento Gonçalves 9090, Bairro Agronomia, Porto Alegre, RS 90540-000, Brasil.*Autor para correspondência: simonepbianchi@gmail.com

${ }^{3}$ Hospital de Clínicas Veterinárias, Universidade Federal do Rio Grande do Sul (UFRGS), Avenida Bento Gonçalves 9090, Bairro Agronomia, Porto Alegre, RS 90540-000.
}

detectadas por histopatologia e por IHQ e uma somente pela IHQ (micrometástase). Uma paciente que apresentava tumor em mamas abdominal caudal e inguinal tinha metástase no linfonodo axilar e inguinal. Assim, observa-se que o tumor pode causar alteração na drenagem linfática provocando metástase em linfonodos que normalmente não drenam determinadas mamas, por isso a retirada do linfonodo axilar deve ser incluída como técnica de rotina para permitir melhor estadiamento das neoplasias mamárias de cadelas.

TERMOS DE INDEXAÇÃO: Linfonodo axilar, neoplasia mamária, cadelas, linfonodo sentinela, patologia.

\section{INTRODUÇÃO}

Neoplasmas mamários são os tumores mais frequentes em cadelas; esses animais devem ser submetidos à estadiamento para definir prognóstico e protocolo terapêutico da doença, assim como é feito em mulheres. Quando submetidas ao procedimento cirúrgico de mastectomia, o linfonodo inguinal por sua posição anatômica é retirado junto da mama 
correspondente. Entretanto o linfonodo axilar somente é retirado quando há alteração na palpação do mesmo. Isso ocorre, em parte, devido à dificuldade de sua localização e pela falta de estudos que comprovem a importância de sua remoção (Cassali et al. 2014).

0 linfonodo sentinela (LNS) é definido como o primeiro gânglio linfático a drenar uma neoplasia, e por esta razão deve ser o primeiro sítio a receber células tumorais se ocorrer disseminação linfática (Paz et al. 2001, Tuohy et al. 2009). Cada vez mais tem sido observado que quando há presença de tumor na cadeia mamária, a drenagem linfática não segue o padrão descrito pela literatura, o que se deve a linfangiogênese estabelecida pela presença do neoplasma. Estudos demonstram que linfonodos correspondentes a regiões que possuem neoplasia mesmo sem apresentar alteração de tamanho e consistência podem já estar com metástase (Beserra et al. 2016).

Foi observado que coletar amostra somente do linfonodo mais próximo ao nódulo não tem sido suficiente para um correto estadiamento tumoral e pode ser negligenciada a presença de doença locorregional (Sonremo et al. 2013). A inspeção do linfonodo regional deve fazer parte da rotina de avaliação dos tumores de mamas em cadelas, pois a presença de metástase tem impacto no estadiamento tumoral do câncer, na sobrevida e no tratamento a ser determinado (Cassali et al. 2014).

Linfonodos podem apresentar metástase (detectadas pela técnica histológica de rotina hematoxilina e eosina) ou micrometástases, o que se refere à disseminação sistêmica de um pequeno número de células tumorais que não são detectadas por histopatologia e que podem ser a grande responsável pela doença metastática em pacientes inicialmente diagnosticados como linfonodo negativo (Tuohy et al. 2009). Em humanos é comum a utilização de citoqueratina para detectar micrometástases ocultas em linfonodos regionais. Ao utilizar essa técnica, é possível avaliar prognóstico relacionado ao intervalo livre de doença e sobrevida global das mulheres submetidas a linfadenectomia (Sorenmo et al. 2011).

Este trabalho teve como objetivo avaliar a importância do linfonodo axilar como sentinela em cadelas com neoplasias mamária submetidas à mastectomia unilateral total.

\section{MATERIAL E MÉTODOS}

Foram tratadas 49 cadelas de diferentes raças, com idade variada, provenientes do atendimento do Hospital de Clínicas Veterinárias da Universidade Federal do Rio Grande do Sul (HCV-FAVET-UFRGS) que apresentavam neoplasia mamária. Esse estudo foi realizado no Hospital de Clínicas Veterinárias da Universidade Federal do Rio Grande do Sul (HCV-UFRGS) e no Laboratório de Anatomia Patológica da FAVET-UFRGS, após aprovação da Comissão de Ética no Uso de Animais da UFRGS (CEUA) sob o número 26411.

Não foram incluídas no estudo cadelas com metástase à distância, que tinham recebido quimioterapia citorredutora prévia ou que possuíssem outra doença concomitante que impossibilitasse a paciente de ser submetida ao procedimento anestésico e cirúrgico. As pacientes não podiam ter sido submetidas a nenhuma cirurgia relacionada às glândulas mamárias.

Com as pacientes já anestesiadas, era aplicado o corante azul patente para marcação linfática e então era removido o linfonodo axilar antes da realização da mastectomia. 0 tempo entre a aplicação do corante e a retirada do linfonodo foi de aproximadamente 10 minutos (figura 1). A definição da técnica cirúrgica de mastectomia unilateral total foi baseada em outro estudo (Stratmann et al. 2008) que mostrou que quando se realiza mastectomia regional, ainda restam $58 \%$ de chance de recidiva do tumor no restante da cadeia mamária ipsilateral.

As amostras (cadeia mamária e linfonodos) coletadas durante o procedimento cirúrgico foram imersas em solução de formalina a $10 \%$ e encaminhadas imediatamente ao Setor de Patologia da Faculdade de Veterinária. Após 24 horas foram processadas pela técnica rotineira de inclusão em bloco de parafina, do qual se obteve corte histológico para coloração de hematoxilina-eosina (HE). Os tumores foram classificados conforme classificação já publicada (Goldschmidt et al. 2011).

As lâminas confeccionadas a partir dos blocos de parafina dos linfonodos inguinal e axilar de cada paciente foram submetidas à técnica de imuno-histoquímica (IHQ) com o anticorpo monoclonal citoqueratina (AE1/AE3) para detecção de células epiteliais metastáticas. Linfonodos foram determinados positivos pela IHQ quando houve marcação de células epiteliais evidenciadas pela cor marrom (figura 2). Todas as lâminas dos tumores primários e dos linfonodos foram avaliadas pelo mesmo patologista.

\section{RESULTADOS}

No total, 49 pacientes tiveram a cadeia mamária e linfonodo(s) avaliados por histopatologia com a coloração de hematoxilina-eosina e IHQ com anticorpo citoqueratina (AE1/AE3). Todas as cadelas realizaram exames pré-cirúrgicos para correto estadiamento tumoral, o que permitiu que oito delas fossem excluídas por presença de metástase à distância. As pacientes que puderam ser incluídas foram submetidas a minucioso exame clínico dos linfonodos pela palpação, no qual somente duas tiveram linfonodos axilares palpáveis no exame clínico.

Os tipos histológicos mais frequentes foram o carcinoma em tumor misto e o carcinoma complexo, com 11 casos cada. Além disso, foram 8 casos de carcinossarcoma, 7 casos de carcinoma e mioepitelioma maligno, 7 carcinomas simples tubulopapilar, dois carcinomas sólidos, um caso de tumor misto benigno, um caso de comedocarcinoma e somente tumor benigno (adenoma de glândula mamária). A mama inguinal foi a que mais apresentou nódulos $(32,65 \%$ dos tumores

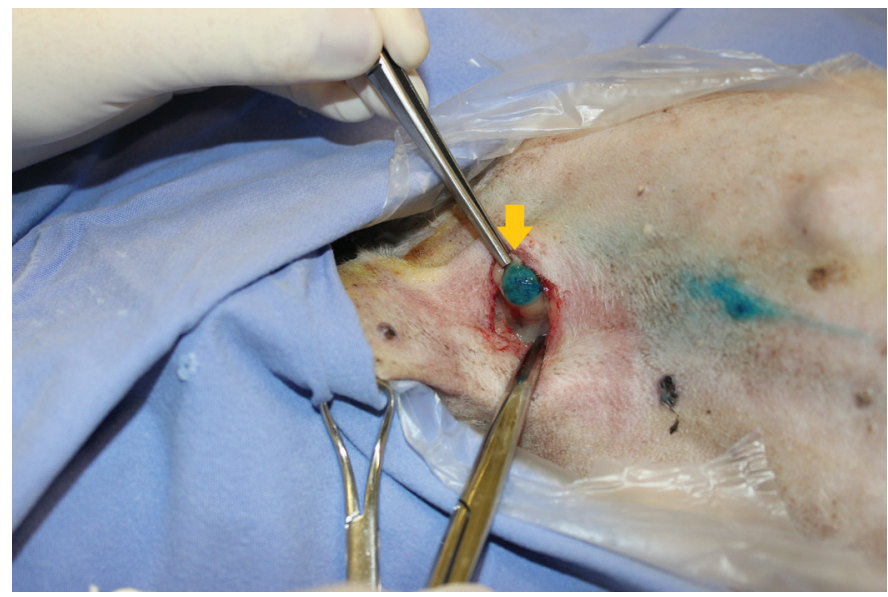

Fig.1. Retirada de linfonodo axilar (seta laranja) em uma cadela com tumor de mama impregnado com corante azul patente $2,5 \%$, o qual foi aplicado em mama torácica cranial por via intradérmica. 

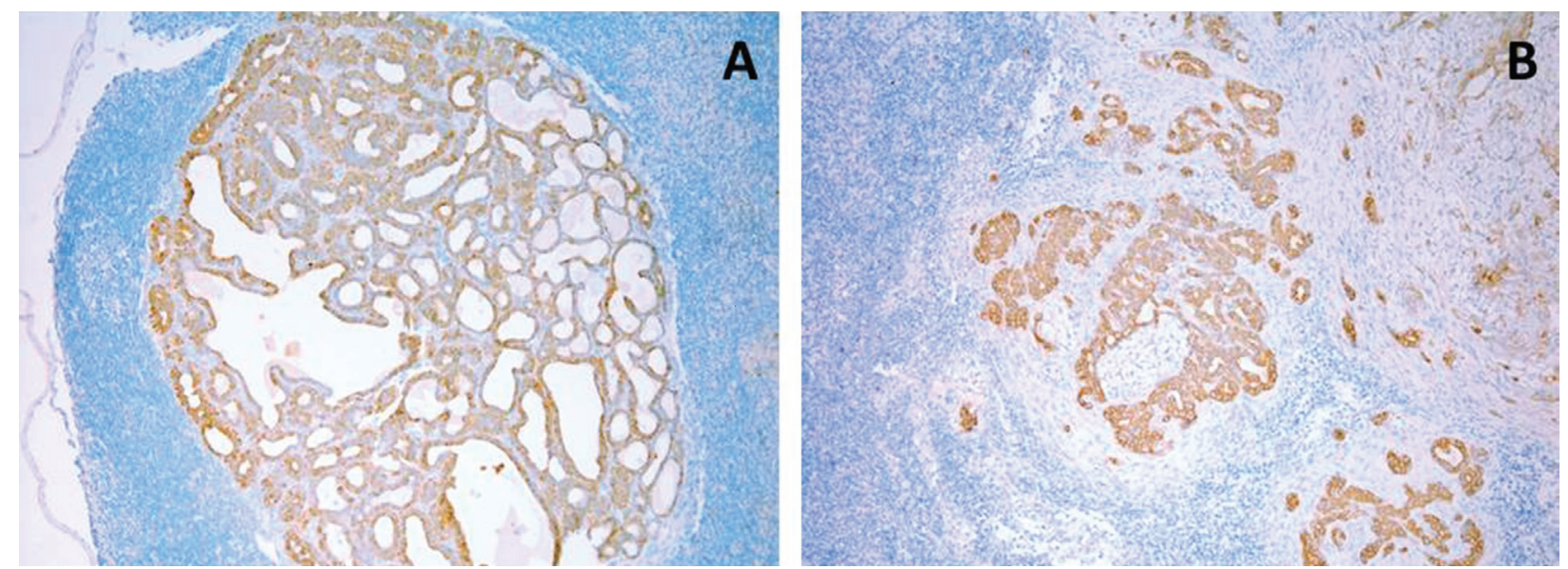

Fig.2. (A,B) Linfonodos de cadelas com tumor de mama após técnica de imuno-histoquímica com anticorpo pancitoqueratina revelando células epiteliais (coradas de marrom).

estavam nessa mama) e apesar disso não foram encontrados muitos casos de metástase em linfonodo inguinal (3 no total).

De todas as cadelas incluídas no estudo, oito das 49 apresentaram linfonodo axilar positivo, dessas sete detectadas por histopatologia e por IHQ imuno-histoquímica e uma somente na IHQ na imuno-histoquímica. No presente estudo, $11(22,45 \%)$ cadelas apresentaram metástase em linfonodo, sendo oito (16,33\%) somente em linfonodo axilar, três em linfonodo inguinal e duas cadelas em ambos.

Foi observado maior número de linfonodos axilares com metástase (8/49) do que de inguinal (5/49) apesar de a neoplasia em mamas caudais ter sido mais frequente. Com relação ao total de cadelas submetidas à avaliação para participar do estudo, 8 de 58 (13,79\%) apresentaram nódulos pulmonares, índice abaixo da metástase em linfonodo, considerando ainda que as 8 pacientes não incluídas no experimento não tiveram seus linfonodos avaliados e não entraram na avaliação final.

Considerando as 49 pacientes incluídas no estudo, o que gerou a análise de 245 mamas (5 por cadela), foi realizada uma correlação entre mama acometida e percentual de nódulos com metástase. Foi observado que na mama torácica cranial (M1) de 11 nódulos observados, 4 (36,4\%) apresentaram metástase. Foi comparado pelo teste Exato de Fisher o percentual de nódulos com metástase da M1 com o percentual nas outras mamas e vemos que essa se destaca por ter uma maior quantidade de nódulos com metástase quando comparada com as outras $(\mathrm{P}=0,011)$. As outras mamas (M2 a M5) não apresentaram diferenças estatisticamente significativas. Uma paciente que apresentava tumor em mamas abdominal caudal e inguinal tinha metástase no linfonodo axilar e inguinal. A maioria das pacientes $(62,5 \%)$ que apresentou metástase em linfonodo axilar tinha nódulos maiores que $3 \mathrm{~cm}$ de diâmetro, e 4/8 (50\%) maiores que $5 \mathrm{~cm}$.

\section{DISCUSSÃO}

Devido a evidência de linfangiogênese nos casos de neoplasma mamário, esse estudo foi focado no interesse de avaliar o percentual de metástase em linfonodo axilar em cadelas submetidas à mastectomia, devido ao fato de o mesmo raramente ser retirado, exceto em caso de alteração na palpação (Tuohy et al. 2009). Foi observado nesse estudo que apenas duas pacientes tinham aumento de tamanho e alteração de consistência do linfonodo axilar na avaliação clínica, mas 8 apresentaram linfonodo axilar com células neoplásicas, o que mostra que a palpação não pode ser utilizada como parâmetro para remoção do linfonodo. Além disso, houve maior percentual de metástase no linfonodo axilar quando o tumor estava nas mamas torácicas, o que sugere que neoplasma nas mamas craniais tende a ser mais agressivos do que nas mamas caudais.

Uma cadela com neoplasia em mama abdominal caudal apresentou metástase nos linfonodos axilar e inguinal. Considerando que essa mama é encontrada próxima ao linfonodo inguinal superficial, não seria realizada a retirada do linfonodo axilar pela técnica de rotina. Entretanto, outro estudo (Pereira et al. 2003) relatou que em cadelas sadias essa mama drena exclusivamente para o linfonodo inguinal superficial, mas em animais com neoplasia mamária pode ser encontrada também comunicação da mesma com o linfonodo axilar, o que facilitaria a ocorrência de metástase para cabeça, pescoço e tórax. Assim como descrito anteriormente (Pereira et al. 2003, Patsikas et al. 2006), este estudo não apresentou pacientes com tumores somente em glândulas mamária inguinal com metástase em linfonodo axilar, mas sugere-se que estudos com maior número de animais sejam feitos para que seja possível determinar se realmente não ocorre essa comunicação.

A imuno-histoquímica demonstrou ser um método válido, pois todas as pacientes com metástase diagnosticada na histopatologia tiveram o resultado confirmado pela presença de células epiteliais no linfonodo através da marcação com o anticorpo citoqueratina, além de mais duas que revelaram ter metástase somente pelo método de imuno-histoquímica (uma em inguinal e uma em axilar). Estudos mostram que a imuno-histoquímica é uma importante ferramenta em patologia mamária para pesquisa de micrometástases em linfonodo sentinela (Salles et al. 2009, Sorenmo et al. 2011). A literatura relata taxas de detecção de micrometástases em $7 \%$ a $42 \%$ em linfonodos axilares de mulheres, dependendo do método de estudo empregado e que aproximadamente $25 \%$ das pacientes linfonodo-negativas desenvolverão recidiva da doença. Acredita-se que a recorrência seria devido às 
metástases que não foram detectadas pelos métodos de rotina e cujos pacientes foram subestadiados (Salles et al. 2009), o que poderia ser extrapolado para pequenos animais, visto que duas pacientes só tiveram a metástase diagnosticada pela imuno-histoquímica. Apesar da alta sensibilidade encontrada, a utilização exclusiva da coloração rotineira de hematoxilina e eosina pode dificultar a identificação de células metastáticas isoladas, conduzindo a diagnósticos falso-negativos (Piato et al. 2008, Beserra et al. 2016)

A correlação da ocorrência de metástase em linfonodo axilar considerando o intervalo de confiança 95\% só foi significativa na mama torácica cranial (M1), a qual das 11 cadelas com neoplasia nessa mama, quatro foram positivas. Isso corresponde a $36,4 \%$ apresentando metástase. 0 teste exato de Fisher mostrou que a mama torácica cranial se destaca estatisticamente em relação à ocorrência de metástase, ou seja, a mama mais próxima do linfonodo axilar foi a com maior índice de metástases. Isso poderia sugerir que as mamas torácicas craniais são mais propensas a desenvolver metástase no linfonodo axilar, devido a sua localização, mas também sugere que neoplasias nessa mama tendem a ser mais agressivas, já que as demais que apresentaram número semelhante de tumores não causaram metástase em tantos animais e o valor não foi estatisticamente significativo. Com os resultados obtidos foi possível observar que o linfonodo axilar, que na maioria às vezes não é retirado apresentou taxa muito maior de metástase do que o linfonodo inguinal, o qual usualmente é removido junto à mama inguinal.

0 fato de a glândula mamária inguinal apresentar maior número de nódulos condiz com a literatura que explica que, por essa glândula geralmente ser a maior de todas da cadeia mamária, consequentemente possui maior suprimento sanguíneo e drenagem linfática (Gómez et al. 2012). Como se trata de uma análise secundária seria necessário um número de pacientes maior para ter um resultado mais confiável, pois não há como prever a positividade de metástase e o cálculo amostral foi realizado conforme o que a rotina clínica e cirúrgica permitia.

A maioria dos linfonodos axilares com células neoplásicas tinham nódulos maiores que $3 \mathrm{~cm}$. Isso poderia interferir na ocorrência da metástase em linfonodo visto que os estudos mostram que tumores maiores de $3 \mathrm{~cm}$ de diâmetro são associados a prognóstico ruim.

Assim como observado nesse estudo, suposições quanto à drenagem linfática com base unicamente na proximidade do tumor primário com o linfonodo nem sempre estão corretas (Tuohy et al. 2009) e o conhecimento da drenagem linfática de glândulas mamarias de cadelas com neoplasia é muito importante para o cirurgião não só para realizar a técnica cirúrgica mais adequada, mas também para a determinação de um prognóstico pós-cirúrgico preciso (Patsikas et al. 2006).

\section{CONCLUSÕES}

A avaliação histopatológica do linfonodo foi essencial para determinar se havia ou não presença de metástase.

A análise imuno-histoquímica revelou ser útil na identificação de micrometástases de origem epitelial, as quais não puderam ser detectadas na técnica de hematoxilina-eosina.

Neoplasias em mamas torácicas craniais se mostraram estatisticamente propensos à maior ocorrência de metástase no linfonodo axilar quando comparados a nódulos em outras mamas.
O neoplasma pode causar alteração na drenagem linfática provocando metástase em linfonodos que normalmente não drenam determinadas mamas; por isso a retirada do linfonodo axilar deve ser incluída como técnica de rotina para permitir melhor estadiamento das neoplasias mamárias de cadelas.

\section{REFERÊNCIAS}

Beserra H.E.O., Grandi F., Dufloth R.M., Pinheiro L.G.P., Miot H.A., Vexenat S.C.O.R. \& Rocha N.S. 2016. Metastasis of mammary carcinoma in bitches: evaluation of the sentinel lymph node technique. Adv. Breast Cancer Res. 5(2):58-65. http://dx.doi.org/10.4236/abcr.2016.52006.

Cassali G.D., Lavalle G.E., Ferreira E., Estrela-Lima A., De Nardi A.B., Ghever C., Sobral R.A., Amorim R.L., Oliveira L.O., Sueiro F.A.R., Beserra H.E.O., Bertagnolli A.C., Gamba C.O., Damasceno K.A., Campos C.B., Araujo M.R., Campos L.C., Monteiro L.N., Nunes F.C., Horta R.S., Reis D.C., Luvizotto M.C.R., Magalhães G.M., Raposo J.B., Ferreira A.M.R., Tanaka N.M., Grandi F., Ubukata R., Batschinski K., Terra E.M., Salvador R.C.L., Jark P.C., Delecrodi J.E.R., Nascimento N.A., Silva D.N., Silva L.P., Ferreira K.C.R.S., Frehse M.S., Di Santis G.W., Silva E.O., Guim T.N., Kerr B., Cintra P.P., Silva F.B.F., Leite J.S., Mello M.F.V., Ferreira M.L.G., Fukumasu H., Salgado B.S. \& Torres R. 2014. Consensus for the diagnosis, prognosis and treatment of canine mammary tumors. Braz. J. Vet. Pathol. 7(2):38-69.

Goldschmidt M., Peña L., Rasotto R. \& Zappulli V. 2011. Classification and grading of canine mammary tumors. Vet. Pathol. 48(1):117-131. http:// dx.doi.org/10.1177/0300985810393258. PMid:21266722.

Gómez B., Ramírez R M. \& Maldonado E J. 2012. Presence of lung metastases in bitches affected by malignant mammary neoplasms in Medellin (Colombia). Revta MVZ Córdoba 17(2):2983-2990. http://dx.doi.org/10.21897/rmvz.232.

Patsikas M.N., Karayannopoulou M., Kaldrymidoy E., Papazoglou L.G., Papadopoulou P.L., Tzegas S.I., Tziris N.E., Kaitzis D.G., Dimitriadis A.S. \& Dessiris A.K. 2006. The lymph drainage of the neoplastic mammary glands in the bitch: a lymphographic study. Anat. Histol. Embryol. 35(4):228-234. http://dx.doi.org/10.1111/j.1439-0264.2005.00664.x. PMid:16836586.

Paz W.A., Paim S.P. \& Mello G.L. 2001. Biópsia de linfonodo sentinela: experiência clínica. Revta Bras. Câncer 47:303-308.

Pereira C.T., Rahal S.C., Balieiro J.C.C. \& Ribeiro A.A. 2003. Lymphatic Drainage on healthy and neoplasic mammary glands in female dogs: can it really be altered? Anat. Histol. Embryol. 32(5):282-290. http://dx.doi. org/10.1046/j.1439-0264.2003.00485.x. PMid:12969028.

Piato J.R.M., Pincerato K.M., Gomes V.C.S., Carvalho F.M., Pinheiro W.S. \& Baracat E.C. 2008. Metástase oculta em linfonodo sentinela no câncer de mama em estádios iniciais. Revta Bras. Ginecol. Obstet. 30(9):432-436. http://dx.doi.org/10.1590/S0100-72032008000900002.

Salles M.A., Cúrcio V.S., Perez A.A., Gomes D.S. \& Gobbi H. 2009. Contribuição da imuno-histoquímica na avaliação de fatores prognósticos e preditivos do câncer de mama e no diagnóstico de lesões mamárias. J. Bras. Patol. Med. Lab. 45(3):213-222. http://dx.doi.org/10.1590/S1676-24442009000300006.

Sorenmo K.U., Rasotto R., Zappulli V. \& Goldschmidt M.H. 2011. Development, anatomy, histology, lymphatic drainage, clinical features, and cell differentiation markers of canine mammary gland neoplasms. Vet. Pathol. 48(1):85-97. http://dx.doi.org/10.1177/0300985810389480. PMid:21147765.

Sonremo K.U., Worley D.R. \& Goldschmidt M.H. 2013. Small Animal Clinical Oncology. Elsevier, St Louis, p.538-556.

Stratmann N., Failing K., Richter A. \& Wehrend A. 2008. Mammary tumor recurrence in bitches after regional mastectomy. Vet. Surg. 37(1):82-86. http://dx.doi.org/10.1111/j.1532-950X.2007.00351.x. PMid:18199060.

Tuohy J.L., Milgram J., Worley D.R. \& Dernell W.S. 2009. A review of sentinel lymph node evaluation and the need for its incorporation into veterinary oncology. Vet. Comp. Oncol. 7(2):81-91. http://dx.doi.org/10.1111/j.14765829.2009.00183.x. PMid:19453362. 